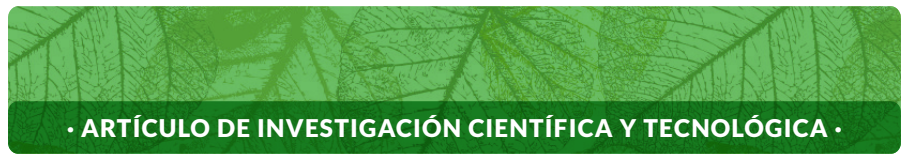

Vol. 20 No 1 · Enero-Junio 2022 · ISSN-1692-3561· ISSN-e 1909-9959· DOI: https://doi.org/10.18684/bsaa.v.n.2022.1746

\title{
Evaluación de la bioaccesibilidad de cadmio en formulaciones de chocolatería por digestión gastrointestinal in vitro*
}

\section{Evaluation of cadmium bioaccessibility in chocolate formulations by in vitro gastrointestinal digestion}

\author{
MURILLO-MÉNDEZ, CRISTIAN-JAHIR ${ }^{1}$; MOLINA-VELASCO, DANIEL-RICARDO²; \\ LÓPEZ-GIRALDO, LUIS-JAVIER ${ }^{3}$; SALAZAR-BELEÑO, ANA-MILENA ${ }^{4}$
}

\section{RESUMEN}

Historial del artículo

Recibido para evaluación: 28 octubre 2020.

Aprobado para publicación: 27 agosto 2021.

* $\quad$ Proyecto de investigación de origen: "Evaluación de la bioaccesibilidad de cadmio en formulaciones de chocolatería por digestión gastrointestinal in vitro”. Financiación: Universidad Industrial de Santander. Culminación: noviembre 2019.

1 Universidad Industrial de Santander, Escuela de Química, Grupo de Investigación en Ciencia y Tecnología de Alimentos (CICTA). M.Sc. Química. Bucaramanga, Colombia. https://orcid.org/0000-0002-5139-0163

2 Universidad Industrial de Santander, Escuela de Química, Laboratorio de Resonancia Magnética Nuclear. Doctor en Química. Bucaramanga, Colombia. https://orcid.org/0000-0002-7897-2526

3 Universidad Industrial de Santander, Escuela de Ingeniería Química, Grupo de Investigación en Ciencia y Tecnología de Alimentos (CICTA). Doctor en Química, Ciencia y Tecnología de Alimentos. Bucaramanga, Colombia. https://orcid.org/0000-0001-9267-7016

4 Instituto Universitario de la Paz (UNIPAZ), Escuela de Ingeniería Agroindustrial, Grupo de Investigación en Innovación, Desarrollo Tecnológico y Competitividad en Sistemas de Producción Agroindustrial (GIADAI). Esp. Aseguramiento de la Calidad e Inocuidad Agroalimentaria. Barrancabermeja, Colombia. https://orcid.org/0000-0001-7592-2550 
Para estudiar la toxicidad del cadmio (Cd) presente en productos de chocolate es necesario conocer su concentración total y determinar la cantidad de Cd que es bioaccesible en el cuerpo humano. Este trabajo se enfocó en evaluar la bioaccesibilidad de $\mathrm{Cd}$ en formulaciones de chocolatería con concentraciones de componentes diferentes por digestión gastrointestinales in vitro. Esta digestión se realizó a través de un titulador automático con control de temperatura y control y monitoreo de $\mathrm{pH}$ mediante soluciones diluidas ácidas y alcalinas. Se correlacionaron las diferencias de bioaccesibilidad con los componentes principales (cacao, leche, azúcar y contenido de $\mathrm{Cd}$ total en el cacao) y, por último, se propuso una formulación de chocolate que tuvo el menor valor de bioaccesibilidad de $\mathrm{Cd}$. Los resultados se expresaron mediante un modelo matemático, con predicciones de $94 \%$ y errores de validación del modelo menores que $9 \%$. Se obtuvieron valores de bioaccesibilidad de Cd en las formulaciones de chocolate entre 7 y $32 \%$ aproximadamente, donde los valores más bajos correspondieron a formulaciones de chocolate amargo (relación másica cacao:azúcar de 7:2). Se concluyó que el contenido de cacao y de azúcar en la formulación disminuyen los valores de bioaccesibilidad de $\mathrm{Cd}$, mientras que el contenido de leche y la concentración de Cd total en el cacao aumentan los valores de bioaccesibilidad.

\section{ABSTRACT}

To study the toxicity of cadmium (Cd) present in chocolate products, it is necessary to know its total concentration and determine the amount of $\mathrm{Cd}$ that is bioaccessible in the human body. This work focused on evaluating the $\mathrm{Cd}$ bioaccessibility in chocolate formulations with concentrations of different components by gastrointestinal digestion in vitro. This digestion was carried out through an automatic titrator with temperature control and $\mathrm{pH}$ control and monitoring using dilute acid and alkaline solutions. Bioaccessibility differences were correlated with the main components (cocoa, milk, sugar, and total $\mathrm{Cd}$ content in cocoa) and, finally, a chocolate formulation was proposed that had the lowest $C d$ bioaccessibility value. The results were expressed using a mathematical model, with predictions of $94 \%$ and model validation errors less than $9 \%$. Cd bioaccessibility values in the chocolate formulations were obtained between 7 and $32 \%$, approximately, where the lowest values corresponded to dark chocolate formulations (cocoa:sugar ratio 7:2). It was concluded that the cocoa and sugar content in the formulation decrease the $\mathrm{Cd}$ bioaccessibility values, while the milk content and the total $\mathrm{Cd}$ concentration in cocoa increase the bioaccessibility values.

Cómo citar este artículo: MURILLO-MÉNDEZ, CRISTIAN-JAHIR; MOLINA-VELASCO, DANIEL-RICARDO; LÓPEZ-GIRALDO, LUIS-JAVIER; SALAZAR-BELEÑO, ANA-MILENA. Evaluación de la bioaccesibilidad de cadmio en formulaciones de chocolatería por digestión gastrointestinal in vitro. Revista Biotecnología en el Sector Agropecuario y Agroindustrial, v. 20, n. 1, 2022, p. 68-83. Doi: https://doi.org/10.18684/bsaa.v.n.2022.1746

\section{PALABRAS CLAVE:}

Cacao; Grasa; Leche; Digestión gástrica; Digestión pancreática; Modelo matemático, Superficie de respuesta; Valor mínimo.

\section{KEYWORDS:}

Cocoa; Fat; Milk; Gastric digestion; Pancreatic digestion; Mathematical model; Response surface; Minimum value. 


\section{INTRODUCCIÓN}

El cacao (Theobroma cacao L.) es un árbol nativo de las regiones tropicales húmedas de la parte norte de Suramérica y de Centroamérica (Motamayor et al., 2002). Posee numerosos componentes bioactivos como polifenoles, que son potentes antioxidantes para la salud humana; metilxantinas y componentes precursores de aroma, en menor proporción (Perea-Villamil et al., 2009; Pico-Hernández et al., 2019; Villamizar-Jaimes \& López-Giraldo, 2017). La producción de cacao es una práctica agrícola que origina productos de exportación importantes para economías en desarrollo de países en África, Asia, Oceanía, Latinoamérica y el Caribe (Jiménez Tobón, 2015), sin embargo, la mayoría del cacao colombiano y, por ende, sus productos derivados como el chocolate, poseen concentraciones elevadas de cadmio.

El cadmio (Cd) es un metal pesado clasificado como contaminante ambiental, proveniente de residuos tanto de fuentes naturales como de prácticas industriales y de agricultura (Hernández-Martínez \& Navarro-Blasco, 2012; Satarug et al., 2017; Alves et al., 2018). Como los metales pesados están presenten en productos alimenticios de consumo humano y animal, el contenido de $\mathrm{Cd}$ es un criterio indispensable a la hora de evaluar los niveles de toxicidad de los alimentos (Alexander et al., 2009; Zhuravlev et al., 2015; Satarug et al., 2017). Se ha determinado su presencia en granos y en productos derivados del cacao, en la mayoría de los casos, la concentración en los granos cultivados en diferentes regiones de Latinoamérica supera el límite que estipula la Unión Europea $(1,0 \mathrm{mg} / \mathrm{kg})$ (European Food Safety Authority, 2011).

Se ha encontrado su presencia en granos de cacao producido en sistemas agroforestales y como de monocultivo, sin diferencias significativas en el contenido (Gramlich et al., 2017). También realizaron la cuantificación con valores promedio superiores al máximo permitido por las normas europeas con 0,9 mg/kg (Chavez et al., 2015; Arévalo-Gardini et al., 2017; Gramlich et al., 2018).

En Colombia, no hay una legislación para el control del Cd en el cacao (Jiménez-Tobón, 2015), pese a esto, altos contenidos podrían limitar la exportación del grano a clientes de la unión europea quienes aprecian los granos de cacao producidos por sus cualidades de fino y de aroma (International Cocoa Organization, 2019). Algunos reportaron concentraciones de 4,05 mg/kg en chocolates amargos (con $65 \%$ de cacao) comprados en supermercados colombianos, valores muy superiores añ límite permitido por la Unión Europea $(0,8 \mathrm{mg} / \mathrm{kg}$ ) (Echeverry \& Reyes, 2016). Sin embargo, algunos productos de chocolate pueden tener concentraciones menores, como por ejemplo el chocolate de mesa en polvo y granulado proveniente de Chiquinquirá, Colombia, que se determinó por voltamperometría diferencial de pulso de redisolución anódica arrojando entre 0,214 y 0,260 mg/kg (Chaparro-Acuña et al., 2017; Lo Dico et al., 2018).

Aunque se ha determinado la cantidad total de Cd en diferentes matrices (Chandra et al., 2014; Zhuang et al., 2016; Bhat et al., 2019), es importante destacar el papel de su bioaccesibilidad, que se define como la cantidad de sustancia que está disuelta en la parte digestiva y aprovechable para absorción (Peixoto et al., 2016), por lo que algunas investigaciones se han enfocado en el estudio de la bioaccesibilidad de los metales pesados para diversas matrices de alimentos, como por ejemplo, los factores que afectan la bioaccesibilidad del $\mathrm{Cd}$ en bebidas achocolatadas donde encontraron que ésta varía según el tipo de muestra, siendo mayor en la formulación light con aproximadamente 11 \% (Peixoto et al., 2016; Francisco et al., 2018). También se encontró que, en concentraciones de sales biliares mayores, la bioaccesibilidad del $\mathrm{Cd}$ aumenta 3 veces más y, tanto el $\mathrm{pH}$ como la presencia de lipasa en la digestión gastrointestinal in vitro, no varía significativamente la bioaccesibilidad del Cd (Peixoto et al., 2016). Barraza y colaboradores determinaron la bioaccesibilidad gástrica humana en grano de cacao crudo y en licor de cacao y obtuvieron valores entre el 90 y $100 \%$ en las digestiones gástricas in vitro para grano y licor de cacao (Barraza et al., 2017).

Algunos autores determinaron la bioaccesibilidad de otros compuestos en productos de cacao, resaltando la selectividad de algunos compuestos en la digestión pancreática (Mounicou et al., 2003), sin embargo, esto no 
sucede con el Cd, donde pueden predominar altos valores de bioaccesibilidad en las fases de digestión, especialmente en la gástrica (Gültekin-Özgüven et al., 2016; Toro-Uribe et al., 2019).

Existen diversos trabajos de investigación en torno a la concentración total de Cd en cacao y sus productos; no obstante, son pocos los trabajos de investigación que estudian la bioaccesibilidad del cadmio en función de los componentes de la matriz, tal es el caso de las matrices como bebida de chocolate en polvo o en grano de cacao.

Por lo anterior, este trabajo se enfocó en el estudio y evaluación del efecto de los componentes principales, comúnmente usados en las formulaciones en chocolate (concentración inicial de cadmio, licor de cacao, azúcar y leche), sobre la bioaccesibilidad de cadmio por medio de digestión gastrointestinal in vitro.

\section{MÉTODO}

\section{Reactivos y muestras}

Las muestras de cacao fermentado y seco se obtuvieron del centro de acopio de cacao en el municipio de San Vicente de Chucurí, uno de los mayores productores en el Departamento de Santander. De allí, se seleccionaron de 6 a 10 muestras, con concentraciones de Cd entre 1 y $10 \mathrm{mg} / \mathrm{kg}$ y a una altitud entre 550 y 1400 m.s.n.m. También, se utilizaron como ingredientes de la preparación leche en polvo y azúcar refinada de marca comercial, libre de Cd. Los reactivos para el procedimiento de digestión gastrointestinal in vitro y la cuantificación de Cd fueron pepsina de mucosa gástrica porcina, pancreatina de páncreas porcino, soluciones estándar de sales biliares, carbonato de amonio y ácido nítrico concentrado; todos marca Merck, mientras que el agua usada en el desarrollo de la investigación fue ultrapura $(0,056 \mu \mathrm{S} / \mathrm{cm})$ tipo 1.

\section{Diseño experimental para el efecto de los factores}

Las coberturas de chocolate para las digestiones gastrointestinales in vitro se fabricaron basado en la Resolución 1511 del INVIMA (Ministerio de la Protección Social, 2011), aplicando un diseño $2^{4}$ más puntos centrales, con cuatro factores que describen los componentes principales de la formulación de cacao (Cuadro 1), con un total de 96 experimentos, teniendo el valor de porcentaje de bioaccesibilidad de $\mathrm{Cd}$ como variable de respuesta. El diseño de experimentos se ejecutó y se analizó mediante el software Statistica.

Cuadro 1. Descripción de los factores y sus niveles para el diseño de experimentos.

\begin{tabular}{|l|c|c|c|}
\hline \multicolumn{1}{|c|}{ Factores } & Nivel mínimo & Nivel máximo & Respuesta \\
\hline Licor de cacao, g & 3 & 7 & \\
\hline Leche en polvo, g & 0 & 4 & \multirow{2}{*}{$\begin{array}{c}\text { \% bioaccesibilidad } \\
\text { de Cd }\end{array}$} \\
\hline Azúcar, $\mathrm{g}$ & 2 & 4 & \\
\hline $\begin{array}{l}\text { Concentración de Cd en } \\
\text { cacao, } \mathrm{mg} / \mathrm{kg}\end{array}$ & 1,23 & 9,11 & \\
\hline
\end{tabular}

\section{Preparación de las formulaciones}

Para la elaboración del licor de cacao, se tostaron los granos de cacao fermentados y secos a una temperatura de $120^{\circ} \mathrm{C}$ durante $20 \mathrm{~min}$. Luego, se retiró la cascarilla del grano de manera manual y se realizó una premolienda durante 2 min a $4^{\circ} \mathrm{C}$. Por último, se refinó el polvo de cacao resultante hasta obtener el licor. Una vez determinadas las cantidades de los ingredientes de las formulaciones por medio de los puntos del diseño experimental, se mezclaron manualmente las proporciones de licor de cacao, leche y azúcar y se refinaron hasta obtener una consistencia líquida y uniforme. Se suprimieron las etapss de atemperado y moldeado dado que solo afectan a la textura del producto. 


\section{Cuantificación de Cd total}

Se cuantificó el contenido de Cd total presente en las coberturas de chocolate con base en el procedimiento propuesto por Echeverry y Reyes (2016). Se calcinaron aproximadamente $3 \mathrm{~g}$ de la muestra en una mufla a 600 ${ }^{\circ} \mathrm{C}$ durante 4 horas. Luego, se disolvieron en $10 \mathrm{~mL}$ de HCl $6 \mathrm{~N}$ y se llevaron a ebullición por $5 \mathrm{~min}$. Por último, se filtró el contenido con papel filtro, se adicionaron 3 gotas de $\mathrm{HNO}_{3}$ al $65 \%$, y se aforó a $25 \mathrm{~mL}$. Las soluciones resultantes se analizaron por Espectrometría de Absorción Atómica (AAS) en llama utilizando un equipo PinAAcle 400 Perkin-Elmer provisto de gas de combustión aire-acetileno a una presión de 2,5 Pa y un flujo de $1 \mathrm{~L} / \mathrm{min}$. La absorbancia de las muestras se leyó directamente a una longitud de onda de $254 \mathrm{~nm}$.

\section{Digestión gastrointestinal in vitro}

Los ensayos de digestión gastrointestinal in vitro se desarrollaron siguiendo el procedimiento descrito por Minekus et al. (2014) con modificaciones. Para la digestión gastrointestinal in vitro, se prepararon soluciones estándar electrolíticas (Cuadro 2) que simularon los fluidos de la digestión gástrica y digestión pancreática usando $\mathrm{NaOH} 1 \mathrm{M} \mathrm{y} \mathrm{HCl} 6 \mathrm{M}$ para ajustar y mantener el $\mathrm{pH}$ de las soluciones.

Cuadro 2. Composición de las soluciones utilizadas como fluidos de las digestiones.

\begin{tabular}{|l|c|c|}
\hline \multirow{2}{*}{ Constituyentes } & $\begin{array}{c}\text { Composición de solución de } \\
\text { fluido gástrico (SFG), mM }\end{array}$ & $\begin{array}{c}\text { Composición de solución de fluido } \\
\text { pancreático (SFP), } \mathrm{mM}\end{array}$ \\
\cline { 2 - 3 } & $\mathbf{p H ~ 3 , 0}$ & $\mathbf{p H ~ 7 , 0}$ \\
\hline $\mathrm{KCl}$ & 6,9 & 6,8 \\
\hline $\mathrm{KH}_{2} \mathrm{PO}_{4}$ & 0,9 & 0,8 \\
\hline $\mathrm{NaHCO}_{3}$ & 25 & 85 \\
\hline $\mathrm{NaCl}$ & 47,2 & 38,4 \\
\hline $\mathrm{MgCl}_{2}\left(\mathrm{H}_{2} \mathrm{O}\right)_{6}$ & 0,1 & 0,33 \\
\hline$\left(\mathrm{NH}_{4}\right) \mathrm{Cl}$ & 0,5 & - \\
\hline
\end{tabular}

Para la digestión gástrica in vitro, se pesaron aproximadamente $5 \mathrm{~g}$ de cada formulación de chocolate y se mezclaron con 7,5 mL de SFG, se adicionaron $1,6 \mathrm{~mL}$ de solución de pepsina porcina de $25000 \mathrm{U} / \mathrm{mL}$ (se disolvió la pepsina en SFG), $5 \mu \mathrm{L}$ de $\mathrm{CaCl}_{2} 0,3 \mathrm{M} \mathrm{y} \mathrm{0,8} \mathrm{mL}$ de HCl $1 \mathrm{M}$ para llegar a $\mathrm{pH}$ 3,0. Todo el procedimiento se realizó durante 2 horas con agitación constante, controlando la temperatura entre 36 y $37^{\circ} \mathrm{C}$ y un valor de $\mathrm{pH}$ entre 2,9 y 3,1. Para la digestión pancreática in vitro, se adicionaron $11 \mathrm{~mL}$ de SFP a la solución resultante de la anterior digestión, luego se adicionaron $5 \mathrm{~mL}$ de solución de pancreatina de $800 \mathrm{U} / \mathrm{mL}$ (se disolvió la pancreatina con SFP), 2,5 mL de bilis al 0,5\%, $40 \mu \mathrm{L}$ de $\mathrm{CaCl}_{2} 0,3 \mathrm{M}, 0,8 \mathrm{~mL}$ de $\mathrm{NaOH} 1 \mathrm{M}$ para llegar a pH 7,0 y 0,3 mL de agua. El procedimiento tuvo una duración de 2 horas con agitación constante, controlando la temperatura entre 36 y $37^{\circ} \mathrm{C}$ y un valor de $\mathrm{pH}$ entre 6,9 y 7,1 .

La determinación del contenido de Cd bioaccesible se basó en la metodología propuesta por Barraza et al. (2017) con modificaciones: se adicionó la solución resultante de la digestión pancreática a un tubo cónico de 50 $\mathrm{mL}$ y se centrifugó a $4000 \mathrm{rpm}$ por $10 \mathrm{~min}$. Luego, se adicionó el sobrenadante a un vaso precipitado de $100 \mathrm{~mL}$, se aagregaron 5 gotas de $\mathrm{HNO}_{3}$ al $65 \%$, se agitó un poco hasta observar un precipitado y se filtró el contenido con papel filtro recogiendo el filtrado en un balón aforado de $50 \mathrm{~mL}$. Por último, se aforó la solución a $50 \mathrm{~mL}$ y se analizó por AAS en llama utilizando como gas de combustión aire-acetileno a las mismas condiciones descritas para la cuantificación de cadmio total. 


\section{Determinación de la bioaccesibilidad del Cd}

Se determinó la bioaccesibilidad del $\mathrm{Cd}\left(\% \mathrm{Cd}_{\text {bio }}\right)$ a partir de la Ecuación 1:

$$
\% C d_{\text {bio }}=\frac{\left[C d_{\text {bioaccesible }}\right]}{\left[C d_{\text {total }}\right]} * 100
$$

Donde [Cd bioaccesible] corresponde al contenido de Cd presente en la solución resultante de la digestión gastrointestinal sobre la cantidad de la formulación de chocolate utilizada en la digestión; y [Cd total] que es la concentración del Cd total de la formulación de chocolate.

\section{Correlación de bioaccesibilidad de cadmio con componentes de formulaciones}

Para determinar la correlación entre los componentes en las formulaciones de chocolate y la bioaccesibilidad de Cd, se usó un ANOVA a los resultados obtenidos. También, se evaluó por medio de superficie de respuesta, diagramas de Pareto y correlación de datos predichos con observados. Se realizó un ajuste de la ecuación que describió el porcentaje de bioaccesibilidad de Cd en función de los factores evaluados que fueron significativos (cacao, azúcar, leche en polvo y concentración de Cd en cacao).

Los datos obtenidos se ajustaron a un modelo matemático de efectos lineales con interacciones como se presenta en la Ecuación 2.

$$
Y=X_{0}+X_{A} A+X_{B} B+X_{C} C+X_{D} D+X_{A B} A B+X_{A C} A C+X_{A D} A D+X_{B C} B C+X_{B D} B D+X_{C D} C D
$$

Donde $\mathrm{Y}$ es el porcentaje de bioaccesibilidad de $\mathrm{Cd}, \mathrm{X}_{\mathrm{n}}$ es el coeficiente de cada variable y A, B, C y D son las variables de cantidad de cacao, leche en polvo, azúcar y concentración de $C$ d en cacao respectivamente.

Finalmente, se procedió a determinar las concentraciones de los componentes de la formulación de chocolate que posea menor valor en la bioaccesibilidad de $\mathrm{Cd}$.

\section{RESULTADOS}

Los resultados mostraron que el valor promedio del porcentaje de bioaccesibilidad de Cd fue de 19,61\% que y los valores máximo y mínimo fueron de 32,56 y 7,22 \% respectivamente (Cuadro 3 ). 
Cuadro 3. Resultados de bioaccesibilidad de cadmio en las formulaciones de chocolate.

\begin{tabular}{|c|c|c|c|c|c|c|c|}
\hline Experimento & Cacao, g & Leche, $\mathrm{g}$ & Azúcar, g & $\begin{array}{c}\text { [Cd] en cacao, } \\
\mathrm{mg} / \mathrm{kg}\end{array}$ & $\begin{array}{c}\text { [Cd] total, } \\
\mathrm{mg} / \mathrm{kg}\end{array}$ & $\begin{array}{c}\text { [Cd] bioaccesible, } \\
\mathrm{mg} / \mathrm{kg}\end{array}$ & $\begin{array}{c}\text { Bioaccesibilidad } \\
\text { de } C d, \%\end{array}$ \\
\hline 1 & 3,00 & 0,00 & 2,00 & 1,23 & 0,74 & 0,14 & 18,92 \\
\hline 2 & 3,00 & 0,00 & 4,00 & 1,23 & 0,53 & 0,10 & 18,87 \\
\hline 3 & 3,00 & 2,00 & 3,00 & 1,23 & 0,46 & 0,10 & 21,74 \\
\hline 4 & 3,00 & 4,00 & 2,00 & 1,23 & 0,41 & 0,10 & 24,39 \\
\hline 5 & 3,00 & 4,00 & 4,00 & 1,23 & 0,34 & 0,08 & 23,53 \\
\hline 6 & 5,00 & 0,00 & 3,00 & 1,23 & 0,77 & 0,10 & 12,99 \\
\hline 7 & 5,00 & 2,00 & 2,00 & 1,23 & 0,69 & 0,12 & 17,39 \\
\hline 8 & 5,00 & 2,00 & 3,00 & 1,23 & 0,63 & 0,11 & 17,46 \\
\hline 9 & 5,00 & 2,00 & 3,00 & 1,23 & 0,63 & 0,12 & 19,05 \\
\hline 10 & 5,00 & 2,00 & 4,00 & 1,23 & 0,58 & 0,10 & 17,24 \\
\hline 11 & 5,00 & 4,00 & 3,00 & 1,23 & 0,53 & 0,12 & 22,64 \\
\hline 12 & 7,00 & 0,00 & 2,00 & 1,23 & 0,97 & 0,07 & 7,22 \\
\hline 13 & 7,00 & 0,00 & 4,00 & 1,23 & 0,80 & 0,08 & 10,00 \\
\hline 14 & 7,00 & 2,00 & 3,00 & 1,23 & 0,71 & 0,09 & 12,68 \\
\hline 15 & 7,00 & 4,00 & 2,00 & 1,23 & 0,66 & 0,13 & 19,70 \\
\hline 16 & 7,00 & 4,00 & 4,00 & 1,23 & 0,58 & 0,09 & 15,52 \\
\hline 17 & 3,00 & 0,00 & 2,00 & 1,23 & 0,75 & 0,14 & 18,67 \\
\hline 18 & 3,00 & 0,00 & 4,00 & 1,23 & 0,54 & 0,11 & 20,19 \\
\hline 19 & 3,00 & 2,00 & 3,00 & 1,23 & 0,46 & 0,10 & 21,74 \\
\hline 20 & 3,00 & 4,00 & 2,00 & 1,23 & 0,42 & 0,10 & 23,81 \\
\hline 21 & 3,00 & 4,00 & 4,00 & 1,23 & 0,34 & 0,07 & 20,59 \\
\hline 22 & 5,00 & 0,00 & 3,00 & 1,23 & 0,77 & 0,10 & 12,99 \\
\hline 23 & 5,00 & 2,00 & 2,00 & 1,23 & 0,67 & 0,12 & 17,91 \\
\hline 24 & 5,00 & 2,00 & 3,00 & 1,23 & 0,62 & 0,10 & 16,13 \\
\hline 25 & 5,00 & 2,00 & 3,00 & 1,23 & 0,62 & 0,12 & 19,35 \\
\hline 26 & 5,00 & 2,00 & 4,00 & 1,23 & 0,56 & 0,11 & 19,64 \\
\hline 27 & 5,00 & 4,00 & 3,00 & 1,23 & 0,52 & 0,12 & 23,08 \\
\hline 28 & 7,00 & 0,00 & 2,00 & 1,23 & 0,94 & 0,07 & 7,45 \\
\hline 29 & 7,00 & 0,00 & 4,00 & 1,23 & 0,77 & 0,08 & 10,39 \\
\hline 30 & 7,00 & 2,00 & 3,00 & 1,23 & 0,72 & 0,09 & 12,50 \\
\hline 31 & 7,00 & 4,00 & 2,00 & 1,23 & 0,66 & 0,12 & 18,18 \\
\hline 32 & 7,00 & 4,00 & 4,00 & 1,23 & 0,57 & 0,10 & 17,54 \\
\hline 33 & 3,00 & 0,00 & 2,00 & 6,63 & 3,98 & 0,87 & 21,86 \\
\hline 34 & 3,00 & 0,00 & 4,00 & 6,63 & 2,84 & 0,62 & 21,83 \\
\hline 35 & 3,00 & 2,00 & 3,00 & 6,63 & 2,49 & 0,67 & 26,91 \\
\hline 36 & 3,00 & 4,00 & 2,00 & 6,63 & 2,21 & 0,69 & 31,22 \\
\hline 37 & 3,00 & 4,00 & 4,00 & 6,63 & 1,81 & 0,45 & 24,86 \\
\hline 38 & 5,00 & 0,00 & 3,00 & 6,63 & 4,14 & 0,69 & 16,67 \\
\hline 39 & 5,00 & 2,00 & 2,00 & 6,63 & 3,68 & 0,80 & 21,74 \\
\hline 40 & 5,00 & 2,00 & 3,00 & 6,63 & 3,32 & 0,70 & 21,08 \\
\hline 41 & 5,00 & 2,00 & 3,00 & 6,63 & 3,32 & 0,70 & 21,08 \\
\hline 42 & 5,00 & 2,00 & 4,00 & 6,63 & 3,01 & 0,64 & 21,26 \\
\hline 43 & 5,00 & 4,00 & 3,00 & 6,63 & 2,76 & 0,73 & 26,45 \\
\hline 44 & 7,00 & 0,00 & 2,00 & 6,63 & 5,16 & 0,53 & 10,27 \\
\hline 45 & 7,00 & 0,00 & 4,00 & 6,63 & 4,22 & 0,52 & 12,32 \\
\hline 46 & 7,00 & 2,00 & 3,00 & 6,63 & 3,87 & 0,58 & 14,99 \\
\hline 47 & 7,00 & 4,00 & 2,00 & 6,63 & 3,57 & 0,80 & 22,41 \\
\hline 48 & 7,00 & 4,00 & 4,00 & 6,63 & 3,09 & 0,60 & 19,42 \\
\hline 49 & 3,00 & 0,00 & 2,00 & 6,63 & 3,98 & 0,88 & 22,11 \\
\hline 50 & 3,00 & 0,00 & 4,00 & 6,63 & 2,84 & 0,64 & 22,54 \\
\hline 51 & 3,00 & 2,00 & 3,00 & 6,63 & 2,49 & 0,65 & 26,10 \\
\hline
\end{tabular}




\begin{tabular}{|c|c|c|c|c|c|c|c|}
\hline Experimento & Cacao, g & Leche, g & Azúcar, g & $\begin{array}{c}\text { [Cd] en cacao, } \\
\mathrm{mg} / \mathrm{kg}\end{array}$ & $\begin{array}{l}\text { [Cd] total, } \\
\mathrm{mg} / \mathrm{kg}\end{array}$ & $\begin{array}{c}\text { [Cd] bioaccesible, } \\
\mathrm{mg} / \mathrm{kg}\end{array}$ & $\begin{array}{c}\text { Bioaccesibilidad } \\
\text { de } C d, \%\end{array}$ \\
\hline 52 & 3,00 & 4,00 & 2,00 & 6,63 & 2,21 & 0,66 & 29,86 \\
\hline 53 & 3,00 & 4,00 & 4,00 & 6,63 & 1,81 & 0,46 & 25,41 \\
\hline 54 & 5,00 & 0,00 & 3,00 & 6,63 & 4,14 & 0,68 & 16,43 \\
\hline 55 & 5,00 & 2,00 & 2,00 & 6,63 & 3,68 & 0,81 & 22,01 \\
\hline 56 & 5,00 & 2,00 & 3,00 & 6,63 & 3,32 & 0,67 & 20,18 \\
\hline 57 & 5,00 & 2,00 & 3,00 & 6,63 & 3,32 & 0,73 & 21,99 \\
\hline 58 & 5,00 & 2,00 & 4,00 & 6,63 & 3,01 & 0,67 & 22,26 \\
\hline 59 & 5,00 & 4,00 & 3,00 & 6,63 & 2,76 & 0,72 & 26,09 \\
\hline 60 & 7,00 & 0,00 & 2,00 & 6,63 & 5,16 & 0,52 & 10,08 \\
\hline 61 & 7,00 & 0,00 & 4,00 & 6,63 & 4,22 & 0,55 & 13,03 \\
\hline 62 & 7,00 & 2,00 & 3,00 & 6,63 & 3,87 & 0,60 & 15,50 \\
\hline 63 & 7,00 & 4,00 & 2,00 & 6,63 & 3,57 & 0,77 & 21,57 \\
\hline 64 & 7,00 & 4,00 & 4,00 & 6,63 & 3,09 & 0,62 & 20,06 \\
\hline 65 & 3,00 & 0,00 & 2,00 & 9,11 & 5,49 & 1,14 & 20,77 \\
\hline 66 & 3,00 & 0,00 & 4,00 & 9,11 & 3,87 & 0,85 & 21,96 \\
\hline 67 & 3,00 & 2,00 & 3,00 & 9,11 & 3,38 & 0,90 & 26,63 \\
\hline 68 & 3,00 & 4,00 & 2,00 & 9,11 & 3,01 & 0,93 & 30,90 \\
\hline 69 & 3,00 & 4,00 & 4,00 & 9,11 & 2,52 & 0,63 & 25,00 \\
\hline 70 & 5,00 & 0,00 & 3,00 & 9,11 & 5,71 & 0,92 & 16,11 \\
\hline 71 & 5,00 & 2,00 & 2,00 & 9,11 & 5,06 & 1,09 & 21,54 \\
\hline 72 & 5,00 & 2,00 & 3,00 & 9,11 & 4,57 & 0,96 & 21,01 \\
\hline 73 & 5,00 & 2,00 & 3,00 & 9,11 & 4,60 & 0,93 & 20,22 \\
\hline 74 & 5,00 & 2,00 & 4,00 & 9,11 & 4,11 & 0,85 & 20,68 \\
\hline 75 & 5,00 & 4,00 & 3,00 & 9,11 & 3,80 & 0,97 & 25,53 \\
\hline 76 & 7,00 & 0,00 & 2,00 & 9,11 & 7,05 & 0,70 & 9,93 \\
\hline 77 & 7,00 & 0,00 & 4,00 & 9,11 & 5,79 & 0,68 & 11,74 \\
\hline 78 & 7,00 & 2,00 & 3,00 & 9,11 & 5,35 & 0,72 & 13,46 \\
\hline 79 & 7,00 & 4,00 & 2,00 & 9,11 & 4,93 & 1,06 & 21,50 \\
\hline 80 & 7,00 & 4,00 & 4,00 & 9,11 & 4,23 & 0,75 & 17,73 \\
\hline 81 & 3,00 & 0,00 & 2,00 & 9,11 & 5,43 & 1,20 & 22,10 \\
\hline 82 & 3,00 & 0,00 & 4,00 & 9,11 & 3,87 & 0,79 & 20,41 \\
\hline 83 & 3,00 & 2,00 & 3,00 & 9,11 & 3,42 & 0,88 & 25,73 \\
\hline 84 & 3,00 & 4,00 & 2,00 & 9,11 & 3,01 & 0,98 & 32,56 \\
\hline 85 & 3,00 & 4,00 & 4,00 & 9,11 & 2,56 & 0,60 & 23,44 \\
\hline 86 & 5,00 & 0,00 & 3,00 & 9,11 & 5,69 & 0,99 & 17,40 \\
\hline 87 & 5,00 & 2,00 & 2,00 & 9,11 & 5,03 & 1,10 & 21,87 \\
\hline 88 & 5,00 & 2,00 & 3,00 & 9,11 & 4,58 & 0,93 & 20,31 \\
\hline 89 & 5,00 & 2,00 & 3,00 & 9,11 & 4,53 & 0,97 & 21,41 \\
\hline 90 & 5,00 & 2,00 & 4,00 & 9,11 & 4,10 & 0,86 & 20,98 \\
\hline 91 & 5,00 & 4,00 & 3,00 & 9,11 & 3,83 & 0,96 & 25,07 \\
\hline 92 & 7,00 & 0,00 & 2,00 & 9,11 & 7,08 & 0,78 & 11,02 \\
\hline 93 & 7,00 & 0,00 & 4,00 & 9,11 & 5,82 & 0,66 & 11,34 \\
\hline 94 & 7,00 & 2,00 & 3,00 & 9,11 & 5,29 & 0,71 & 13,42 \\
\hline 95 & 7,00 & 4,00 & 2,00 & 9,11 & 4,89 & 1,11 & 22,70 \\
\hline 96 & 7,00 & 4,00 & 4,00 & 9,11 & 4,26 & 0,80 & 18,78 \\
\hline
\end{tabular}

Estos valores muestran la influencia de la formulación sobre el porcentaje de bioaccesibilidad de Cd; por tal motivo, es importante determinar las cantidades de cacao, leche y azúcar, y la concentración de Cd en el cacao adecuadas para la elaboración de formulaciones de chocolate con menores valores de porcentaje de bioaccesibilidad.

Se aplicó un ANOVA a los valores obtenidos con la ecuación 2 para comparar la significancia de las variables en el modelo (Cuadro 4). Al comparar el valor $p$ de cada variable se puede apreciar que solo la interacción BD, que corresponde a la cantidad de leche y a concentración de Cd en el cacao, no tiene efecto significativo (valor 
$p>0,05)$. Asimismo, se observa que las variables correspondientes a la cantidad de cacao y de leche son las que mayor influencia posee en el modelo inicial propuesto, debido a su valor $F$.

Cuadro 4. Resultado ANOVA de las variables del modelo inicial planteado.

\begin{tabular}{|l|c|c|c|}
\hline \multicolumn{1}{|c|}{ Factor } & Suma de cuadrados (SS) & Valor $\mathbf{F}$ & Valor $\mathbf{P}$ \\
\hline A: Cacao, g & 1181,112 & 718,2899 & 0,000000 \\
\hline B: Leche, g & 863,688 & 525,2495 & 0,000000 \\
\hline C: Azúcar, g & 13,846 & 8,4206 & 0,004723 \\
\hline D: $[$ Cd] cacao, $\mathrm{mg} / \mathrm{kg}$ & 178,712 & 108,6829 & 0,000000 \\
\hline AB & 42,150 & 25,6334 & 0,000002 \\
\hline AC & 12,383 & 7,5307 & 0,007399 \\
\hline AD & 7,295 & 4,4363 & 0,038131 \\
\hline BC & 77,877 & 47,3607 & 0,000000 \\
\hline BD & 2,494 & 1,5169 & 0,221486 \\
\hline CD & 11,962 & 7,2745 & 0,008432 \\
\hline Error & 139,769 & & \\
\hline Total SS & 2622,307 & & \\
\hline
\end{tabular}

Los efectos estandarizados del modelo inicial se pueden observar en el diagrama de Pareto (Figura 1) en el que se visualiza que las variables cantidad de cacao y de leche son las de mayor inciden en el modelo. Sin embargo, la cantidad de cacao (que posee la mayor influencia) tiene un efecto negativo en el porcentaje bioaccesibilidad de $\mathrm{Cd}$, mientras que la cantidad de leche y la concentración de Cd en cacao posee un efecto positivo, observándose en el signo negativo y positivo de cada efecto estandarizado, respectivamente (Figura 1).

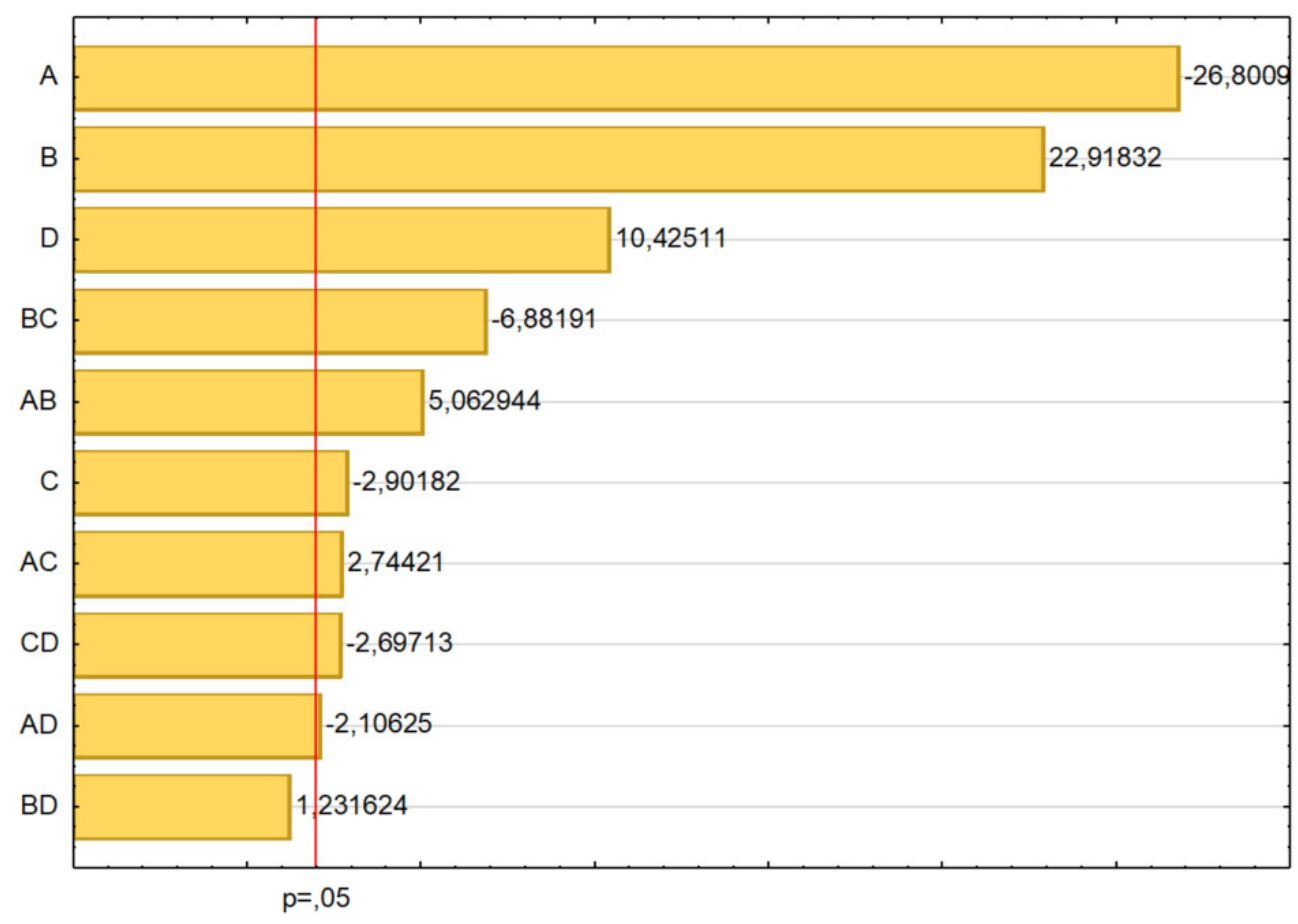

Standardized Effect Estimate (Absolute Value)

Figura 1. Diagrama de Pareto con los valores absolutos de los efectos estandarizados estimados para el modelo inicial planteado en la ecuación. Las variables A, B, C y D corresponden a cantidad de cacao, leche en polvo, azúcar y concentración de $\mathrm{C}$ en cacao respectivamente. 


\section{Efecto de la cantidad de cacao}

Según los resultados, la cantidad de cacao en la formulación de chocolate aporta en el contenido de Cd total, por lo tanto, a mayor cantidad de cacao utilizado, mayor contenido de Cd total. Siguiendo la misma lógica, se esperaría que, a mayor cantidad de cacao, mayor contenido de $\mathrm{Cd}$ en la fase bioaccesible después de la digestión gastrointestinal; sin embargo, este efecto es contrario. Una de las razones por las que se presenta este efecto es por la presencia de grasa en el cacao: la presencia de componentes no polares (grasa) impide la liberación de metales y moléculas polares en la fase polar y disminuye la probabilidad de ser bioaccesibles después de la digestión gastrointestinal (Martini et al., 2018). Además, en la digestión pancreática hay presencia de bilis, que contribuye a la disolución y degradación de las grasas y, por ende, puede producir compuestos más pequeños que pueden atrapar metales o moléculas polares de menor tamaño en forma de micelas o complejos insolubles en la digestión. Esto disminuye la cantidad de Cd libre en la solución restante de la digestión y disminuye la concentración de Cd bioaccesible después de la digestión gastrointestinal (Peixoto et al., 2016; Barraza et al., 2017).

\section{Efecto de la cantidad de leche}

Teniendo en cuenta la cantidad de leche en polvo que se utilizó para la elaboración del producto, a medida que este valor incrementa, el porcentaje de bioaccesibilidad también incrementa. Se corroboró que la leche en polvo no contribuye al contenido de Cd total a la formulación, sin embargo, el contenido de aminoácidos polares y de ácidos orgánicos es elevado. La presencia de un componente polar de gran proporción en las formulaciones de chocolate como la leche y, sumado a esto, el alto contenido de acidez presente en ella ayuda a liberar más iones de $\mathrm{Cd}^{2+}$ en la fase polar y aumentar la probabilidad de ser bioaccesible después de la digestión gastrointestinal (Cobbett \& Goldsbrough, 2002).

\section{Efecto de la cantidad de azúcar}

A medida que la cantidad de azúcar usada en las formulaciones se incrementó, teniendo constantes las demás variables de la formulación, el porcentaje de bioaccesibilidad disminuyó. Aunque el efecto de la cantidad de azúcar es pequeño en comparación con el efecto de las otras variables (Figura 1), ésta es una variable significativa en el modelo inicial, lo que puede ser atribuido a la formación de complejos de C insolubles con la sacarosa y otros endulzantes en la fase intestinal de la digestión, reduciendo la concentración de Cd bioaccesible y, por ende, el porcentaje de bioaccesibilidad de Cd (Peixoto et al., 2016).

\section{Efecto de la concentración de Cd en el cacao}

Al incrementar la concentración de $\mathrm{C} d$ en el cacao, el porcentaje de bioaccesibilidad también incrementa, debido a que la matriz posee mayor cantidad de $\mathrm{Cd}$ que puede ser solubilizada y ser bioaccesible en las digestiones gastrointestinales.

\section{Modelo matemático}

De acuerdo con lo anterior, los datos se ajustaron a un nuevo modelo corregido de efectos lineales con interacciones, eliminando la interacción BD (cantidad de leche y [Cd] en cacao) la cual no fue significativa (Ecuación 3):

$$
Y=24,92-3,20 A+2,67 B+0,22 C+1,09 D+0,23 A B+0,25 A C-0,05 A D-0,64 B C-0,14 C D
$$

El modelo ajustado permitió explicar el comportamiento de los datos experimentales en un 94,01 \%, observándose una tendencia lineal entre los datos experimentales y los obtenidos con el modelo ajustado (Figura 2). 


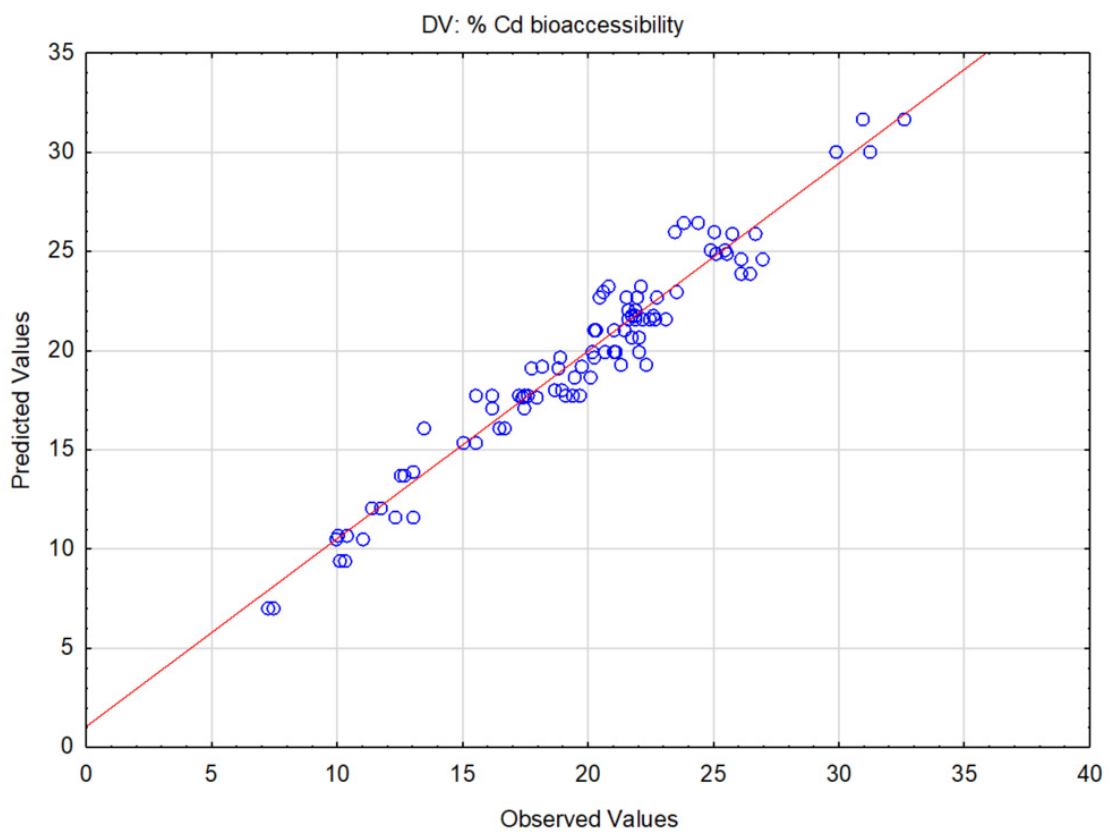

Figura 2. Valores observados con respecto a valores predichos con el modelo ajustado para el porcentaje bioaccesibilidad. de Cd.

En la superficie de respuesta se puede observar una tendencia de los datos similares a la reportada en la gráfica de Pareto, con una disminución del porcentaje bioaccesibilidad Cd cuando la cantidad de cacao aumenta, y un aumento en el porcentaje bioaccesibilidad cuando las cantidades de leche aumentan (Figura 3).

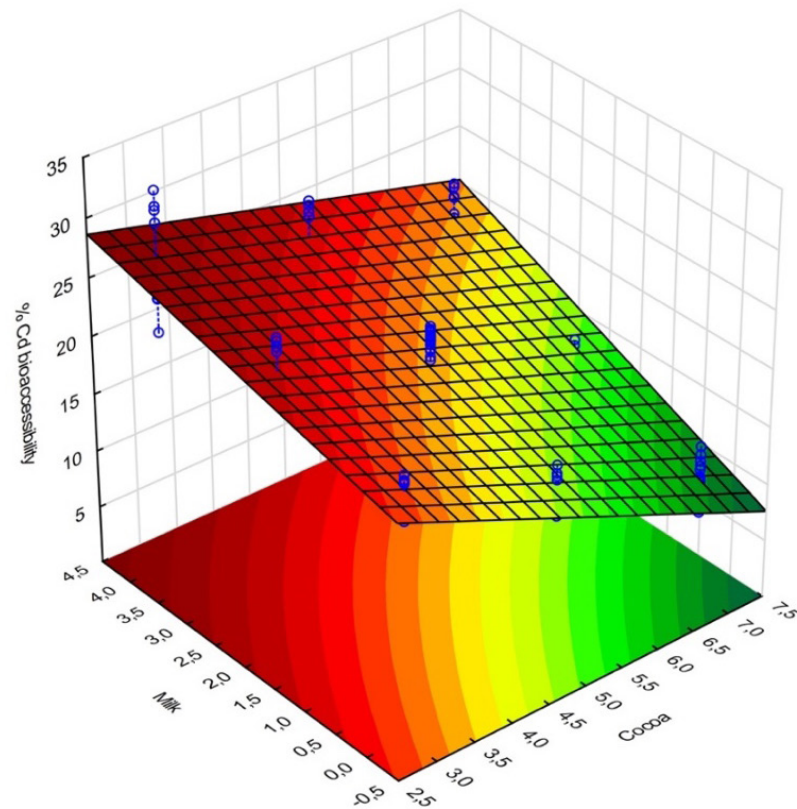

Figura 3. Superficie de respuesta del modelo ajustado graficando \% de bioaccesibilidad de $\mathrm{Cd}$ en función de cantidad de leche y cacao, manteniendo las otras dos variables fijas a mitad del valor. 


\section{Valor mínimo de bioaccesibilidad}

Una vez obtenido el modelo matemático para describir el comportamiento del porcentaje bioaccesibilidad de $\mathrm{Cd}$ en las formulaciones de chocolate, se obtuvieron los valores de las variables que corresponden a formulaciones con el valor mínimo de bioaccesibilidad de $\mathrm{Cd}$ de 7,07 \%, donde se obtuvo una formulación con $7 \mathrm{~g}$ de cacao, $0 \mathrm{~g}$ de leche y $2 \mathrm{~g}$ de azúcar, utilizando un cacao de $1,23 \mathrm{mg} / \mathrm{kg}$ de Cd. Así mismo, otros valores mínimos se obtuvieron para formulaciones de chocolate con otras concentraciones de $\mathrm{Cd}$ en el cacao y mayor cantidad (Cuadro 5). También, se obtuvo el valor experimental de la formulación de chocolate propuesta por el modelo ajustado, obteniéndose un error de $2,08 \%$.

Cuadro 5. Puntos experimentales de valor mínimo de porcentaje de bioaccesibilidad de Cd utilizando cacao de [Cd] conocida.

\begin{tabular}{|c|c|c|c|c|c|c|}
\hline \multirow{2}{*}{ Cacao, $\mathbf{g}$} & \multirow{2}{*}{ Leche, $\mathbf{g}$} & \multirow{2}{*}{ Azúcar, $\mathbf{g}$} & \multirow{2}{*}{$\begin{array}{c}\text { [Cd] en cacao, } \\
\mathbf{m g} / \mathbf{k g}\end{array}$} & \multicolumn{2}{|c|}{ Bioaccesibilidad de Cd, \% } & \multirow{2}{*}{ Error, \% } \\
\cline { 5 - 7 } & & & 1,23 & 7,22 & 7,07 & 2,08 \\
\hline 7 & 0 & 2 & 5,43 & 9,21 & 8,94 & 2,96 \\
\hline 7 & 0 & 2 & 11,65 & 10,57 & 9,24 \\
\hline 7 & 0 & 2 & 9,11 & Valor experimental & \\
\hline
\end{tabular}

Estos resultados muestran que las formulaciones de chocolate, para cualquier concentración de Cd del cacao utilizado dentro del intervalo del modelo (Cuadro 1), que poseen menor valor de porcentaje de bioaccesibilidad de Cd corresponden a chocolate negro o amargo (dark chocolate) donde el alto contenido de grasa puede favorecer a la retención del $\mathrm{Cd}$ en la digestión gastrointestinal, y la ausencia de compuestos ácidos, como la leche, puede evitar la liberación del Cd en su forma bioaccesible.

\section{Evaluación del modelo}

La evaluación del modelo ajustado se adelantó con formulaciones diferentes a los puntos experimentales utilizados para el modelo, se calculó el error del valor prestablecido por el modelo para 10 formulaciones experimentales donde se obtuvieron errores de 8,84\% en promedio (Cuadro 6).

Cuadro 6. Puntos experimentales de formulaciones de chocolate para la validación del modelo ajustado.

\begin{tabular}{|c|c|c|c|c|c|c|}
\hline \multirow{2}{*}{ Cacao, g } & \multirow{2}{*}{ Leche, $\mathrm{g}$} & \multirow{2}{*}{ Azúcar, g } & \multirow{2}{*}{$\begin{array}{c}\text { [Cd] en cacao, } \\
\mathrm{mg} / \mathrm{kg}\end{array}$} & \multicolumn{2}{|c|}{ Bioaccesibilidad de Cd, \% } & \multirow{2}{*}{ Error, \% } \\
\hline & & & & Valor experimental & Valor predicho & \\
\hline 3 & 0 & 3 & 9,11 & 23,27 & 22,97 & 1,31 \\
\hline 3 & 2 & 2 & 6,63 & 28,51 & 25,80 & 9,51 \\
\hline 3 & 2 & 4 & 9,11 & 27,33 & 24,26 & 11,23 \\
\hline 5 & 0 & 2 & 6,63 & 16,47 & 15,57 & 5,45 \\
\hline 5 & 0 & 4 & 6,63 & 18,73 & 16,66 & 11,09 \\
\hline 5 & 4 & 4 & 9,11 & 24,54 & 22,39 & 8,77 \\
\hline 70 & 0 & 30 & 6,63 & 11,64 & 10,55 & 9,31 \\
\hline 70 & 20 & 40 & 6,63 & 16,87 & 15,03 & 10,86 \\
\hline 70 & 40 & 30 & 6,63 & 22,02 & 19,99 & 9,22 \\
\hline 3087 & 0 & 1023 & 8,41 & 15,22 & 13,45 & 11,64 \\
\hline
\end{tabular}

El modelo se ajustó para las variables, sin embargo, el modelo puede aplicarse para grandes cantidades de cacao, leche y azúcar (A, B y $C$ respectivamente) siempre y cuando se mantengan las proporciones estipuladas para este modelo (Cuadro 1). Esta afirmación se sustenta en resultados obtenidos (Cuadro 6) para formulaciones de mayor masa; por ejemplo, para una formulación de $4 \mathrm{~kg}$, los valores experimental y presteblecido de bioaccesibilidad de Cd fueron 15,22 y 13,45\%, respectivamente, diferencia que representa tan solo un 11,64\% de error. 


\section{Comparación con otros valores}

Los valores obtenidos de porcentaje de bioaccesibilidad de Cd para esta investigación se compararon con resultados reportados por otros autores (Cuadro 7). Si bien, las matrices utilizadas en los otros trabajos no son las mismas, utilizan como base cacao en los productos elaborados.

Cuadro 7. Comparación del porcentaje de bioaccesibilidad de Cd con otros autores.

\begin{tabular}{|l|c|c|c|}
\hline \multirow{2}{*}{ Valor } & \multicolumn{3}{|c|}{ Bioaccesibilidad de Cd, \% } \\
\cline { 2 - 4 } & $\begin{array}{c}\text { Chocolate } \\
\text { (Este trabajo) }\end{array}$ & $\begin{array}{c}\text { Licor de cacao } \\
\text { (Barraza et al. 2017) }\end{array}$ & $\begin{array}{c}\text { Polvo de bebida de chocolate } \\
\text { (Peixoto et al. 2016) }\end{array}$ \\
\hline Promedio & $19,61 \pm 5,25$ & $99,2 \pm 4,13$ & $6,5 \pm 3,04$ \\
\hline Menor & 7,22 & 91,0 & 3,0 \\
\hline Mayor & 32,56 & 106,2 & 11,1 \\
\hline
\end{tabular}

Una de las diferencias en el valor de porcentaje de bioaccesibilidad de Cd de este trabajo con el trabajo de Barraza y colaboradores es debido a que solo consideraron la digestión gástrica como procedimiento para determinar la fracción de $\mathrm{Cd}$ bioaccesible. Una de las razones de esta consideración es que ellos mencionan la probabilidad de absorción de $\mathrm{Cd}$ antes de entrar a la fase pancreática, pero es una hipótesis aún por confirmar (Barraza et al., 2017). También, modificaron las condiciones de la digestión para obtener altos porcentajes de bioaccesibilidad y maximizar los efectos de una posible intoxicación (Barraza et al., 2017).

Los valores de bioaccesibilidad del trabajo de Peixoto y colaboradores son menores a los reportados ya que ellos utilizaron diferentes formulaciones de polvo de bebida, algunas de las cuales con adiciones de compuestos funcionales que contribuyeron a una reducción del porcentaje bioaccesibilidad de $\mathrm{Cd}$. Además, optimizaron la cantidad de bilis y el $\mathrm{pH}$ en las digestiones gastrointestinales, incluyendo adiciones de compuestos dietarios como pectina y celulosa, que condujeron a menores valores de bioaccesibilidad de Cd (Peixoto et al., 2016).

\section{CONCLUSIONES}

Se obtuvo un modelo matemático para correlacionar el porcentaje de bioaccesibilidad de cadmio con los componentes cacao, leche, azúcar y concentración de $\mathrm{C}$ d total en el cacao; donde explicó el comportamiento de los datos experimentales con los datos prestablecidos en un 94,01\% y la evaluación del modelo mostró errores de 8,84\%, en promedio. En el modelo se encontró efecto significativo en todas las contribuciones lineales, observándose efectos negativos en la cantidad de cacao y azúcar, y efectos positivos en la cantidad de leche y la concentración de Cd en cacao. La contribución lineal de la cantidad de cacao y la cantidad de leche fueron las variables más significativas en el modelo. Se propuso una formulación con $77,8 \%$ de cacao y $22,2 \%$ de azúcar, que corresponde a un chocolate amargo, con un porcentaje de bioaccesibilidad de Cd de 7,07\%. Además, utilizando cacao de concentración de $\mathrm{Cd}$ dentro del intervalo del modelo, se propusieron formulaciones que corresponde a chocolate amargo (dark chocolate) que poseen porcentaje de bioaccesibilidad de $\mathrm{Cd}$ menores al $11 \%$. Los presentes resultados orientan al productor y comercializador a conocer las formulaciones de cacao pertinentes a disminuir la exposición y toxicidad de Cd, con granos de cacao que posean alto contenido de $\mathrm{Cd}$ provenientes de regiones del Santander. 


\section{REFERENCIAS}

ABT, EILEEN; SAM, JENNIFER-FONG; GRAY, PATRICK; ROBIN, LAUREN POSNICK. Cadmium and lead in cocoa powder and chocolate products in the US Market. Food Additives \& Contaminants: Part B, v. 11, n. 2, 2018, p. 92-102. https://doi.org/10.1080/19393210.2017.1420700

ABT, EILEEN; ROBIN, LAUREN-POSNICK. Perspective on Cadmium and Lead in Cocoa and Chocolate. Journal of Agricultural and Food Chemistry, v. 68, n. 46, 2020, p. 13008-13015. https://dx.doi.org/10.1021/acs.jafc.9b08295

ALVES, RICARDO N.; MAULVAULT, ANA L.; BARBOSA, VERA L.; FERNANDEZ-TEJEDOR, MARGARITA; TEDIOSI, ALICE; KOTTERMAN, MICHIEL; VAN DEN HEUVEL, FREDERICUS H.M.; ROBBENS, JOHAN; FERNANDES, JOSÉ O.; RASMUSSEN, RIE-ROMME; SLOTH, JENS J.; MARQUES, ANTÓNIO. Oral bioaccessibility of toxic and essential elements in raw and cooked commercial seafood species available in European markets. Food Chemistry, v. 267, 2018, p. 15-27. https://doi.org/10.1016/j.foodchem.2017.11.045

ARÉVALO-GARDINI, ENRIQUE; ARÉVALO-HERNÁNDEZ, CESAR O.; BALIGAR, VIRUPAX C.; HE, ZHENLI L. Heavy metal accumulation in leaves and beans of cacao (Theobroma cacao L.) in major cacao growing regions in Peru. Science of The Total Environment, v. 605-606, 2017, p. 792-800. http://dx.doi.org/10.1016/j.scitotenv.2017.06.122

BARRAZA, F.; SCHRECK, E.; LÉVEQUE, T.; UZU, G.; LÓPEZ, F.; RUALES, J.; PRUNIER, J.; MARQUET, A.; MAURICE, L. Cadmium bioaccumulation and gastric bioaccessibility in cacao: A field study in areas impacted by oil activities in Ecuador. Environmental Pollution, v. 229, 2017, p. 950-963. http://dx.doi.org/10.1016/j.envpol.2017.07.080

BARRAZA, FIORELLA; SCHRECK, EVA; UZU, GAËLLE; LÉVEQUE, THIBAUT; ZOUITEN, CYRIL; BOIDOT, MATHIEU; MAURICE, LAURENCE. Beyond cadmium accumulation: Distribution of other trace elements in soils and cacao beans in Ecuador. Environmental Research, v. 192, 2021, p. 110241. https://doi.org/10.1016/j.envres.2020.110241

BHAT, ZUHAIB F.; MORTON, JAMES D.; MASON, SUSAN L.; BEKHIT, ALAA EL-DIN A.; MUNGUREB, TANYARADZWA E. Pulsed electric field: Effect on in-vitro simulated gastrointestinal protein digestion of deer Longissimus dorsi. Food Research International, v. 120, 2018, p. 793-799. https://doi.org/10.1016/j.foodres.2018.11.040

CHAPARRO-ACUÑA, SANDRA-PATRICIA; VARGAS-MORENO, PAOLA-ANDREA; SILVA-GÓMEZ, LEONARDO-ANDRÉS; CÁRDENAS, OSWALDO-ELIÉCER. Cadmium voltametric quantification in table chocolate produced in Chiquinquira-Boyaca, Colombia. Acta Agronómica, v. 66, n. 2, 2017, p. 172-177. http://dx.doi.org/10.15446/acag.v66n2.5847

DE OLIVEIRA, VINICIUS-HENRIQUE; ULLAH, IHSAN; DUNWELL, JIM M.; TIBBETT, MARK. Bioremediation potential of $\mathrm{Cd}$ by transgenic yeast expressing a metallothionein gene from Populus trichocarpa. Ecotoxicology and Environmental Safety, v. 202, 2020, p. 110917. https://doi.org/10.1016/j.ecoenv.2020.110917

ECHEVERRY, A.; REYES, H. Determinación de la concentración de cadmio en un chocolate colombiano con 65 $\%$ de cacao y chocolates extranjeros con diferentes porcentajes de cacao. Entre Ciencia e Ingeniería, v. 19, 2016, p. 22-32.

https://revistas.ucp.edu.co/index.php/entrecienciaeingenieria/article/view/452

FEBRIANTO, NOOR-ARIEFANDIE; XHU, FAN. Changes in the Composition of Methylxanthines, Polyphenols, and Volatiles and Sensory Profiles of Cocoa Beans from the Sul 1 Genotype Affected by Fermentation. Journal of Agricultural and Food Chemistry, v. 68, n. 32, 2020, p. 8658-8675.

https://dx.doi.org/10.1021/acs.jafc.0c02909 
FRANCISCO, JOÃO; CARDOSO, CARLOS; BANDARRA, NARCISA; BRITO, PEDRO; HORTA, ANDRÉ; PEDROSA, RUI; GIL, MARIA M.; DELGADO, INÉS-MARGARIDA; CASTANHEIRA, ISABEL; AFONSO, CLÁUDIA. Bioaccessibility of target essential elements and contaminants from Fucus spiralis. Journal of Food Composition and Analysis, v. 74, 2018, p. 10-17. https://doi.org/10.1016/j.jfca.2018.08.003

GRAMLICH, A.; TANDY, S.; ANDRES, C.; CHINCHEROS-PANIAGUA, J.; ARMENGOT, L.; SCHNEIDER, M.; SCHULIN, R. Cadmium uptake by cocoa trees in agroforestry and monoculture systems under conventional and organic management. Science of The Total Environment, v. 580, 2017, p. 677-686. http://dx.doi.org/10.1016/j.scitotenv.2016.12.014

GRAMLICH, A.; TANDY, S.; GAUGGEL, C.; LÓPEZ, M.; PERLA, D.; GONZALEZ, V.; SCHULIN, R. Soil cadmium uptake by cocoa in Honduras. Science of The Total Environment, v. 612, 2018, p. 370-378. http://dx.doi.org/10.1016/j.scitotenv.2017.08.145

GÜLTEKIN-ÖZGÜVEN, MINE; BERKTAŞ, IJLAL; ÖZÇELIK, BERAAT. Change in stability of procyanidins, antioxidant capacity and in-vitro bioaccessibility during processing of cocoa powder from cocoa beans. LWT-Food Science and Technology, v. 72, 2016, p. 559-565. https://doi.org/10.1016/j.Iwt.2016.04.065

HERNÁNDEZ-MARTÍNEZ, RAQUEL; NAVARRO-BLASCO, IÑIGO. Estimation of dietary intake and content of lead and cadmium in infant cereals marketed in Spain. Food Control, v. 26, n. 1, 2012, p. 6-14. https://doi.org/10.1016/j.foodcont.2011.12.024

INTERNATIONAL COCOA ORGANIZATION. Quarterly Bulletin of Cocoa Statistics Cocoa Year 2016/17. Quarterly Bulletin of Cocoa Statistics, v. 43, n. 1, 2019, p. 1-147.

KASA, NURSU-AYLIN; BÜYÜKPINAR, ÇAĞDAŞ; ERULAŞ, AHMET-FATIH; BAKIRDERE, SEZGIN. A sensitive and accurate analytical method for the determination of cadmium in food samples: Molybdenum coated T-shape slotted quartz tube flame atomic absorption spectrophotometry. Food Chemistry, v. 319, 2020, p. 126572. https://doi.org/10.1016/j.foodchem.2020.126572

LEWIS, CALEB; LENNON, ADRIAN M.; EUDOXIE, GAIUS; SIVAPATHAM, PARAMASIVAM; UMAHARAN, PATHMANATHAN. Plant metal concentrations in Theobroma cacao as affected by soil metal availability in different soil types. Chemosphere, v. 262, 2021, p. 127749. https://doi.org/10.1016/j.chemosphere.2020.127749

LO DICO, GIANLUIGI-MARIA; GALVANO, FABIO; DUGO, GIACOMO; D'ASCENZI, CARLO; MACALUSO, ANDREA; VELLA, ANTONIO; GIANGROSSO, GIUSEPPE; CAMMILERI, GAETANO; FERRANTELLI, VINCENZO. Toxic metal levels in cocoa powder and chocolate by ICP-MS method after microwaveassisted digestion. Food Chemistry, v. 245, 2018, p. 1163-1168. https://doi.org/10.1016/j.foodchem.2017.11.052

MARTINI, SERENA; CONTE, ANGELA; TAGLIAZUCCHI, DAVIDE. Bioaccessibility, bioactivity and cell metabolism of dark chocolate phenolic compounds after in vitro gastro-intestinal digestion. Journal of Functional Foods, v. 49, 2018, p. 424-436. https://doi.org/10.1016/j.jff.2018.09.005

MINEKUS, M.; ALMINGER, M.; ALVITO, P.; BALANCE, S. A standardised static in vitro digestion method suitable for food - an international consensus. Food \& Function, v. 5, n. 6, 2014, p. 1113-1124. https://doi.org/10.1039/C3FO60702J

MOUNICOU, S.; SZPUNAR, J.; ANDREY, D.; BLAKE, C.; LOBINSKI, R. Concentrations and bioavailability of cadmium and lead in cocoa powder and related products. Food Additives and Contaminants, v. 20, n. 4, 2003, p. 343-352. http://dx.doi.org/10.1080/0265203031000077888

PEIXOTO, RAFAELLA R.A.; DEVESA, VICENTA; VÉLEZ, DINORAZ; CERVERA, M. LUISA; CADOREA, SOLANGE. Study of the factors influencing the bioaccessibility of 10 elements from chocolate drink powder. Journal of Food Composition and Analysis, v. 48, 2016, p. 41-47.

http://dx.doi.org/10.1016/j.jfca.2016.02.002 
PEIXOTO, RAFAELLA R.A.; OLIVEIRA, ANDREA; CADORE, SOLANGE. Risk assessment of cadmium and chromium from chocolate powder. Food Additives \& Contaminants: Part B, v. 11, n. 4, 2018, p. 256-263. https://doi.org/10.1080/19393210.2018.1499676

PICO-HERNÁNDEZ, SERGIO M.; JAIMES-ESTÉVEZ, JAIME; LÓPEZ-GIRALDO, LUIS J.; MURILLO-MÉNDEZ, CRISTIAN J. Supercritical extraction of bioactive compounds from Cocoa husk: study of the main parameters. Revista Facultad de Ingeniería Universidad de Antioquia, v. 91, 2019, p. 95-105. http://dx.doi.org/10.17533/udea.redin.n91a09

RECKNAGEL, SEBASTIAN; KOCH, MATTHIAS; KÖPPEN, ROBERT; BUTTLER, SABINE; PENK, SIBYLLE; MAUCH, TATJANA; SOMMERFELD, THOMAS; WITT, ANGELIKA. Development of certified reference materials for the determination of cadmium and acrylamide in cocoa. Analytical and Bioanalytical Chemistry, v. 412, n. 19, 2020, p. 4659-4668. https://doi.org/10.1007/s00216-020-02719-0

SATARUG, SOISUNGWAN; VESEY, DAVID A.; GOBE, GLENDA C. Current health risk assessment practice for dietary cadmium: Data from different countries. Food and Chemical Toxicology, v. 106, 2017, p. 430-445. https://doi.org/10.1016/j.fct.2017.06.013

TORO-URIBE, SAID; LÓPEZ-GIRALDO, LUIS-JAVIER; ALVAREZ-RIVERA, GERARDO; IBÁÑEZ, ELENA; HERRERO, MIGUEL. Insight of Stability of Procyanidins in Free and Liposomal Form under an in Vitro Digestion Model: Study of Bioaccessibility, Kinetic Release Profile, Degradation, and Antioxidant Activity. Journal of Agricultural and Food Chemistry, v. 67, n. 7, 2019, p. 1990-2003. https://pubs.acs.org/doi/10.1021/acs.jafc.9b00351

VILLAMIZAR-JAIMES, ARLEY-RENÉ; LÓPEZ-GIRALDO, LUIS-JAVIER. Cáscara de cacao fuente de polifenoles y fibra: simulación de una planta piloto para su extracción. Respuestas, v. 22, n. 1, 2017, p. 75-83. https://doi.org/10.22463/0122820X.821

ZHUANG, PING; ZHANG, CHAOSHENG; LI, YINGWEN; ZOU, BI; MO, HUI; WU, KEJUN; WU, JINGTAO; LI, ZHIAN. Assessment of influences of cooking on cadmium and arsenic bioaccessibility in rice, using an in vitro physiologically-based extraction test. Food Chemistry, v. 213, 2016, p. 206-214.

http://dx.doi.org/10.1016/j.foodchem.2016.06.066 